\title{
Adaptive Filtering Technique for Chronic Wound Analysis under Tele-Wound Network
}

\author{
Chinmay Chakraborty* and Bharat Gupta \\ Department of Electronics and Communication, Birla Institute of Technology, \\ Jharkhand, India \\ *Corresponding Author: cchakrabarty@bitmesra.ac.in
}

Received 2 March 2016; Accepted 21 March 2016;

Publication 16 September 2016

\begin{abstract}
Efficient diagnosis of chronic wound depends on the quality of digital image that has reached the Tele-Medical Hub through Tele-Wound Network. However, how much ever precaution we take, while capturing the image by digital camera or by smartphone, presence of random/impulse noise would be always there to corrupt the captured image. The wound images give the vital information such as size, wound status, tissue composition and healing rate. In this paper, we have proposed adaptive filtering technique for chronic wound $(\mathrm{CW})$ image analysis under Tele-wound network to improve the diagnosis. Here, the best filter has been chosen which can help to improve the diagnosis of wound. A comparative study of 16 different filters has also been performed on 72 different wound images. The experimental results are given by comparing 8 different parameters. These various parameters are Peak Signal to Noise Ratio (PSNR), Mean Square Error (MSE), Signal to Noise Ratio (SNR), Negative Absolute Error (NAE), Maximum Difference (MD), Mean Structural Similarity Index (MSSIM), Universal Image Quality Index (UIQI) and Mean Absolute Error (MAE). Simulated results shows adaptive median provides better performances with respect to high value of PSNR (66.23), SNR (58.05) and lower value of MSE (3.01), MAE (0.29), and NAE (0.01) between original and filtered image. The proposed methodology will assist the clinicians to take better decision towards diagnosis of $\mathrm{CW}$ in terms of qualitative at low-resource setup.
\end{abstract}

Journal of Communication, Navigation, Sensing and Services, Vol. 1, 57-76.

doi: 10.13052/jconasense2246-2120.2016.005

(C) 2016 River Publishers. All rights reserved. 
Keywords: Adaptive Median Filter; Chronic Wound; Wound Image Analysis; Tele-Wound Network.

\section{Introduction}

Tele-wound network (TWN) is defined as the delivery of medical health care services and sharing of clinical information over a distance using telecommunication platform $[1,2]$. The objective is to provide better healthcare service to any place wherever and whenever required. TWN system offers reliable and efficient diagnostic aids to patients at affordable cost. TWN allows new technologies in sensing, medical imaging and wireless data communications with much lower cost, enabling the development of new widespread remote medicine initiatives. Wound healing process is highly depend on time i.e. may be required lengthy and several appointments with a clinician for treatment. TWN would reduce the problem of repeated and timely visits to doctors by remote monitoring and controlling. TWN would minimize the burden of health care unit and decreases the patients' worries. Efficient screening of CW images is important for prognostic and therapeutic intervention. Wound healing takes longer duration and regular check-up in certain interval is one of the biggest hinderance in wound caring and healing [1]. Visual assessment by medical experts is time-consuming, subjective and inconsistent whereas computerized analysis of wound images will be rapid and efficient approach; which requires appropriate image processing techniques and experts' domain knowledge for improved screening. A CW not only affects the individual but entire family gets suffered due to the enormous cost of wound care. Furthermore, it should be noted that without intensive medical care and treatment, a CW will not heal most probable. The consequences of chronic wounds are mainly delayed to repair, suspended skin re-growth, slow older cell proliferation rate, social isolation, depression, decrease in productivity and costly treatment. There are 5 types of open wounds viz. abrasion (topmost layer of the skin is removed; painful), incision (smooth edges), laceration (irregular edges), puncture (deep; narrow wound) and avulsion (bleed heavily). The color change of CW's gives important implication of the wound healing status. The image noise is the variation of color information randomly in images produced by image sensor with smartphones and digital camera. Hence, proper filtering is required to remove the random or impulse noise from wound images. The morphological filtering is used to preserve edge information of an image and eliminating the noise [3]. Wild et al. [4] developed digital wound measurement system where quality of the pictures has been maintained by filter of the device. 
An optical imaging system is used in TWN system for capturing skin wound images from the patient. Skin's abnormal conditions are common in the developing countries where symptoms of skin diseases are observed [1]. Christos et al. [5] has been taken 77 color wound photographs through Nikon digital SLR camera and used polarized filters to reduce the light reflection from CW surface. Lie et al. [6] captured wound images by the smartphone's camera and used Gaussian smoothing filter to minimize the noise. Barone et al. [7] used SCIELab spatial filters for original wound image. Ederson et al. [8] applied anisotropic diffusion filtering to avoid the undesirable effects on segmentation for dermatological ulcers. Marina et al. [9] used difference of Gaussians (DoG) filter for wound segmentation. In the help of DoG filtering, local intensity variations has been equalized. Dorra et al. [10] used Gabor filtering technique for multispectral imaging. In this paper, the objective is to investigate best filter amongst all used filters on the basis of image quality assessment. The camera images mostly suffer from independent impulse noise that can be introduced on a wound image may be random/impulse noise. The best filtering technique is required to remove the noises for better quality of image. The adaptive wound image pre-processing module has been developed using adaptive median filter. This proposed TWN system model will automatically check the optimal value of critical parameters and based on that it will select the best filtering techniques.

The organization of paper is as follows: Section 2 describes the system architecture of TWN system. In Section 3, different types of filters are discussed in respect of wound image processing. Section 4 deals with experimental results and discussion based on the image quality parameter. Conclusion is discussed in Section 5.

\section{Tele-Wound Network System Architecture}

In India, major percentage of the population is living in rural areas. They have been facing many problems due to shortages of healthcare facilities. Easy and affordable connectivity is required in rural areas to provide healthcare. Smartphone's integrated with TWM system have the potential to provide costeffective treatment to rural area. The patients' outcomes can be improved; support's user mobility; reduced hospitalization; manage extensive medical data; provides continuity care; minimize the risk; reducing healthcare cost and time using this framework. TWN system model has been proposed for this case could be efficiently used in remote location where specialists' services are not readily available. 
TWN system is used for characterizing wound tissue accurately and deals with large number of wound affected patients. The portable, handheld device like smartphones can be used to capture high-quality wound images and acquire patient's demographic information. These devices would be able to send information to the Tele-Medical Hub (TMH) [11, 12] through 2G, 2.5G (iDEN, GPRS, EDGE), 3G (WCDMA, CDMA2000, TD-CDMA), WLAN and $4 \mathrm{G}$ technologies where the provision of faster data rate is possible. Wound healing process is time depended i.e. it may required lengthy and several appointments with a clinician. Chronic wound image analysis under TWN system scenario could help in cut down the time required. Hence, high quality wound images are required to diagnosis the wound status. Quality of wound image can be enhanced at pre-processing stage by using various filtering techniques. Figure 1 represents the TWN system for wound status monitoring. The processed wound image goes to image processing toolbox in TMH for pre-processing purpose. The adaptive wound image pre-processing

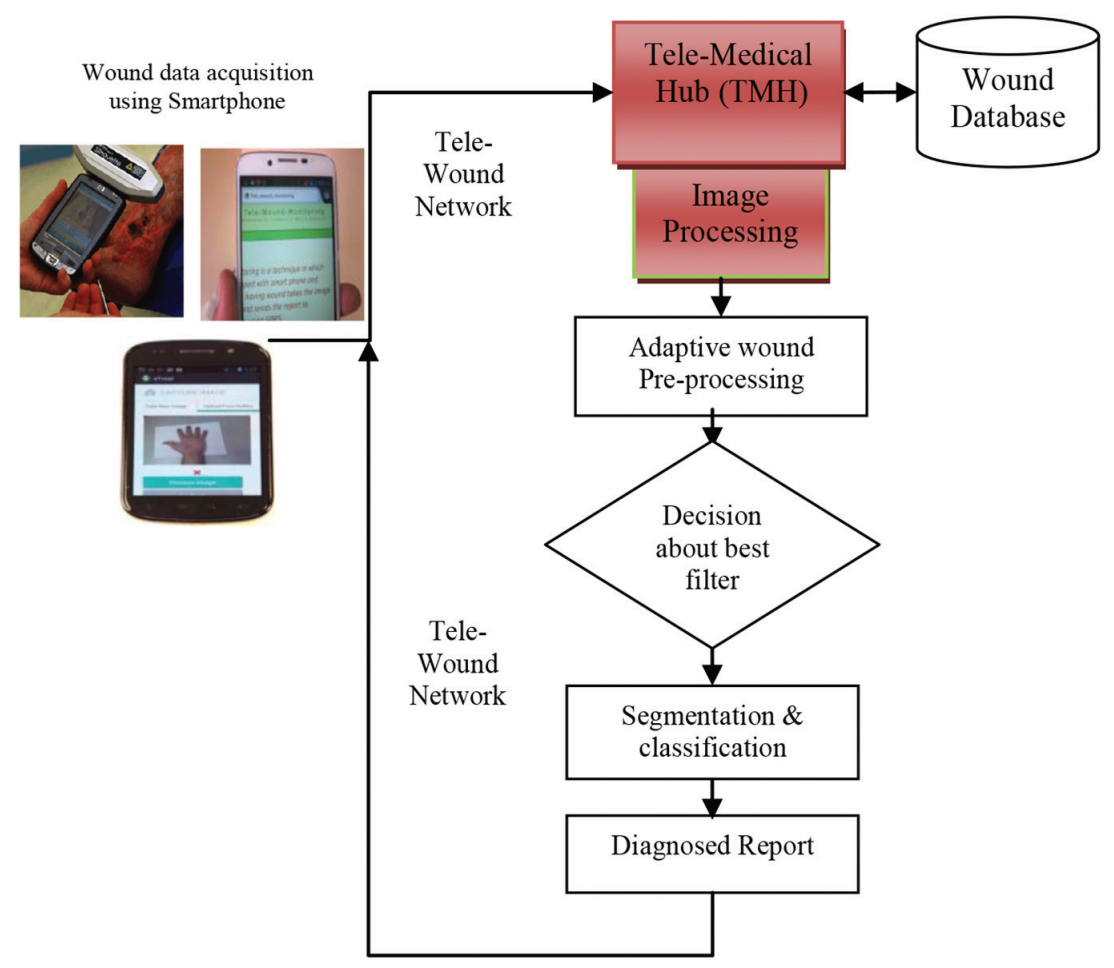

Figure 1 Tele-wound network (TWN) system. 
module has been introduced in this system architecture to improve the quality of image. The patient clinical or demographic information has been stored in wound database. The wound image filtering technique is required to remove the random/impulse noise. The wound image is smoothed by adaptive median filtering for noise reduction by providing well-defined and continuous edge information. Wireless communication technology enabled TWN system reduces the need to transport patients for physical consultation from the expert doctors which avoids the physical and mental stress, reduces time and cost. This framework is very much effective for both rural and urban people; it provides good performance in terms of wound monitoring and advanced diagnostic.

\section{Denoising Filters for Wound Imaging}

The image denoising is a substantial medical imaging task that is able to remove noise while preserving edges. In this paper, sixteen different filters have been considered and a comparative study is presented below. Figure 2 depicts the schematic diagram for wound image pre-processing with adaptive filtering technique.

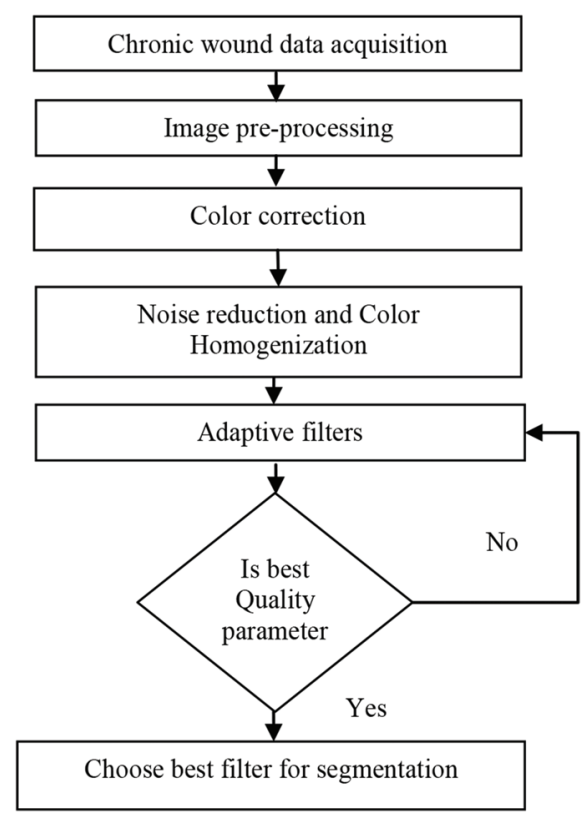

Figure 2 Schematic diagram for wound image processing. 
These important filters are as follows: Mean, Average, Min, M3, Max, Log, Laplacian, Midpoint, Gaussian, Contra Harmonic, Median, Butterworth, Weiner, Alpha Trim Mean, Gabor, and Adaptive Median. Explanation of each filter technique is given below:

\subsection{Mean Filter}

This filter is belongs to linear filtering scheme. This filtering is used to smoothing the images, mainly reducing the intensity variation between pixels. It is useful for removing Gaussian noise but preserving criteria is poor. The statistical model of the noise is swown in below:

$$
\begin{aligned}
p(x) & =\frac{1}{\sigma \sqrt{2 \pi}} e^{-\frac{(x-\mu)^{2}}{2 \sigma^{2}}} \\
\text { Mean filter }\left(x_{1} \ldots x_{N}\right) & =\frac{1}{N} \sum_{i=1}^{N} x_{i}
\end{aligned}
$$

Where, $x_{1} \ldots \ldots x_{N}$ is the pixel range of image, $\sigma$ is the standard deviation and $N$ is the mask size.

\subsection{Average Filter}

It is non-linear smoothing filter. This filter is used to avoids the edge blurring by searching the homognity of current pixel neighbourhood and sharpend resulting image.

\subsection{Min Filter}

This filter is used to fine darkest point in an image and also reduces the salt noise.

$$
\text { Min filter } \hat{f}(x, y)=\min _{(s, t) \in S_{x, y}}[g(s, t)]
$$

Where, $f(x, y)$ is represented as filtered image, $g(s, t)$ is the original corrupted image and window size depicted as $s_{x, y}$.

\subsection{M3 Filter}

This is the smoothing and hybrid of mean and median filter and used to remove the noise.

$$
M 3 \text { filter } f(x, y)=\max \left[\underset{(s, t) \in S_{x y}}{\operatorname{mean}}\{g(s, t)\}, \underset{(s, t) \in S_{x y}}{\operatorname{median}}\{g(s, t)\}\right]
$$


Where, $f(x, y)$ is represented as filtered image, $g(s, t)$ is the original corrupted image and window size depicted as $s_{x, y}$.

\subsection{Max Filter}

This filter is used to findout the brightest point in an image and reduces the pepper noise from the wound image.

$$
\text { Max filter } \hat{f}(x, y)=\max _{(s, t) \in S_{x y}}\{g(s, t)\}
$$

Where, $f(x, y)$ is represented as filtered image and $g(s, t)$ is the original corrupted image.

\subsection{Log Filter}

This filter is used to smoothing the wound image and it is very sensitive to noise. This is a formula for LoG (combination of Laplacian and Gaussian functions) filtering:

$$
\log (x, y)=-\frac{1}{\pi \sigma^{4}}\left[1-\frac{x^{2}+y^{2}}{2 \sigma^{2}}\right] e^{-\frac{x^{2}+y^{2}}{2 \sigma^{2}}}
$$

Where $\sigma$ is the standard deviation.

\subsection{Laplacian Filter}

It is used to sharpening and calculates the second derivatives of an image and also measures the rate of change the first derivatives.

$$
\text { Laplacian filter } g(x, y)=f(x, y)+c\left[\nabla^{2} f(x, y)\right]
$$

Where, $f(x, y)$ is represented as filtered image, $g(x, y)$ is the original corrupted image and $\nabla$ represents the $2^{\text {nd }}$ derivate respectively.

\subsection{Midpoint Filter}

This filter is very much useful for gaussian and uniform noise. This is the combination of order and mean filter. It is the average of highest pixel and the lowest pixel with respect to intensity within the window $s_{x, y}$. So, the outcome of re instated wound image $f$ at point $(x, y)$ is given by,

$$
\text { Midpoint filter } \hat{f}(x, y)=1 / 2\left[\max _{(s, t) \in S_{x, y}}\{g(s, t)\}+\min _{(s, t) \in S_{x, y}}\{g(s, t)\}\right]
$$


Where, $f(x, y)$ is represented as filtered image and $g(s, t)$ is the original corrupted image.

\subsection{Gaussian Filter}

This popular filter is responsible for corrects the spectral coefficient and aplitude spectrum officients within filter window. Gaussian filter is similar to mean filter, but uses different kernel that depcts the shape of a Gaussian. In one dimension, the Gaussian function can be represented as:

$$
G(x)=\frac{1}{\sqrt{2 \pi \sigma}} e^{-\frac{x^{2}}{2 \sigma^{2}}}
$$

Where $\sigma$ is the standard deviation of the distribution.

\subsection{Median Filter}

The Median filter is used in pre-processing stage for providing the image color identification. The bright white spot arises on the wound image due to reflection. Median filter with $5 \times 5$ window size is employed to eliminate the random/impulse noise in wound images [13]. The median filter can smooth noisy regions, enhance the low-contrast wound region and better preserve the structure of the edges. It considers each image pixel and looks at its close by neighbors to choose whether or not it is representative of its environs. Median filter replaces the value of central pixel with the median of all the surrounding neighborhood pixel values. The median is intended by first categorization the entire pixel values from the neighboring neighborhood into numerical categorize and then restoring the pixel being measured with the middle pixel value. The average of two middle pixel values is taken when the neighborhood under consideration contains an even number of pixels. The Median filter is represented as:

$$
\text { Median filter }\left(x_{1} \ldots x_{N}\right)=\text { Median }\left[\left\|x_{1}\right\|^{2} \ldots \ldots\left\|x_{N}\right\|^{2}\right]
$$

The two major advantages of median filter [14] over mean filter are: (a) the median is more robust averages than mean and as such, a single very unrepresentative pixel in a neighborhood will not significantly affect the median value and (b) as the median value is actually the value of one of the pixels in the neighborhood, it does not generate new improbable pixel values when the filter straddles an edge. Hence, the median filter is much superior at preserving sharp edges than mean filter. 


\subsection{Adaptive Median Filter}

Adaptive median filtering has been widely used as an advanced technique compared with standard median filtering. This is performs spatial processing to determine which pixels in a wound image have been affected by random/impulse noise. This filter classifies pixels as noise by comparing every pixel in the image to its surrounding neighbor pixels. The noise pixels are replaced by median pixel value in the neighborhood. This filter removes impulse noise with higher probabilities and reduces distortion along the edges.

\subsection{Contra Harmonic Filter}

This is used to remove the Gaussian noise and preserve edge features.

\subsection{Butterworth Filter}

This filter is the sum of low pass filters in frequency domain where pass band are designed to have a fixed gain with no ripples until the cut-off frequency.

\subsection{Weiner Filter}

It is used to produce an estimate of a desired random process by linear timeinvariant filtering of an observed noisy process. This filter is designed for a desired frequency response and also used for restoration of a degraded image.

\subsection{Alpha Trim Mean Filter}

This filter is used for restoration of signals and wound images corrupted by non-Gaussian noise. It is hybrid of linear and nonlinear approaches.

\subsection{Gabor Filter}

This filter can be used to remove the noise efficiently. It responds to edges and texture changes.

\subsubsection{Color Homogenization}

CWs are having complex mixture of colored tissues with varied intensity. Anisotropic diffusion filtering [15] is used for homogenization of color along with preservation of edges, only smoothing between edges. The objective of this method is to reduce image noise without eliminating significant portion of the image. The color homogenization is used in this work using combined Gray world assumption and Retinex theory [16]. 


\section{Result and Discussion}

In this section, we present result of sixteen denoising filters. The outcomes of the different filters are shown in Figure 11. The pre-processing stage is implemented to improve the efficiency using color correction, noise filtering, and color homogenization methods for improving the wound image quality. Color is the important indication for the visual $\mathrm{CW}$ tissue assessment. This visual red-yellow-black (R-Y-B) color evaluation model has been used mostly in clinical trials on wound assessment. The color corrected RGB image is then assessed for different filters. All 16 filters were applied on set of 72 randomly selected wound images from our developed wound database as well as an online wound image database. Most of them use mathematical morphology operations. In order to access the quality of image after filtering are based on 8 different quantitative performance measures, such as PSNR, MSE, SNR, NAE, MD, MSSIM, UIQI and MAE.

\subsection{MSE}

It is the most important image quality measuring method. It is basically squared error between enhanced image and original CW image. Mathematically, defined as:

$$
M S E=\frac{1}{M \times N} \sum_{x=0}^{M-1} \sum_{y=0}^{N-1}\left[F(x, y)-F_{f l t r}(x, y)\right]^{2}
$$

where, $F(x, y)$ and $F_{f l t r}(x, y)$ are the input, filtered output images and size $M \times N$.

\subsection{PSNR}

It is used to calculate the ratio between maximum possible intensity value of the CW image and MSE. PSNR is defined as:

$$
P S N R=10 \log _{10}\left[\frac{R^{2}}{\frac{1}{M \times N} \sum_{x=0}^{M-1} \sum_{y=0}^{N-1}\left[F(x, y)-F_{f l t r}(x, y)\right]^{2}}\right]
$$

Here, $R$ is the maximum possible peak intensity value of the image. 


\subsection{SNR}

It is the ratio between signal i.e. enhanced image and noise in the spatial domain. In this case, noise is the difference between input image and filtered image. SNR can be computed as:

$$
S N R=\left[\frac{\sum_{x=0}^{M-1} \sum_{y=0}^{N-1}\left[F_{f l t r}(x, y)\right]^{2}}{\sum_{x=0}^{M-1} \sum_{y=0}^{N-1}\left[F(x, y)-F_{f l t r}(x, y)\right]^{2}}\right]
$$

Where, $F(x, y)$ and $F_{f l t r}(x, y)$ are the input and filtered output images.

\subsection{MAE}

This method is used to measure how close predictions are to the eventual outcomes. The MAE is given by

$$
M A E=\frac{1}{n} \sum_{i=1}^{n}\left|f_{i}-y_{i}\right|=\frac{1}{n} \sum_{i=1}^{n}\left|e_{i}\right|
$$

where, MAE is an average of the absolute errors, $e_{i}=\left|f_{i}-y_{i}\right|, f_{i}$ is the prediction and $y_{i}$ is the true value, $n$ is the number of observations.

\subsection{MSSIM}

This method is used to predict the quality of CW images. It is designed to improve the PSNR and MSE values [17].

$$
\operatorname{SSIM}_{(x, y)}=\frac{\left(2 \mu_{x} \mu_{y}+c_{1}\right)\left(2 \sigma_{x y}+c_{2}\right)}{\left(\mu_{x}^{2}+\mu_{y}^{2}+c_{1}\right)\left(\sigma_{x}^{2}+\sigma_{y}^{2}+c_{2}\right)}
$$

Where, $\mu_{x}$ and $\mu_{y}$ is the average of $x$ and $y, \sigma_{x}^{2}$ and $\sigma_{y}^{2}$ is the variance of $x$ and $y, \sigma_{x y}$ is the covariance of $x$ and $y$ respectively.

\subsection{NAE}

NAE [3] is used to find out the similarity between original and filtered image i.e. larger value of NAE provides poor quality of filtered image. 


$$
N A E=\frac{\sum_{x=1}^{M} \sum_{y=1}^{N}\left|F(x, y)-F_{f i l t r}(x, y)\right|}{\sum_{x=1}^{M} \sum_{y=1}^{N}|F(x, y)|}
$$

\subsection{MD}

MD [18] measures the maximum difference in the intensity values of original wound image and filtered image.

$$
M D=\max \left|F_{f i l t r}(x, y)-F(x, y)\right|
$$

\subsection{UIQI}

This parameter can be modeled using three important factors viz. loss of correlation, luminance distortion and contrast distortion.

$$
U I Q I=\frac{1}{i} \sum_{k=1}^{i} Q_{k}
$$

Where,

$$
Q_{k}=\frac{\sigma_{i, f_{g}}}{\sigma_{i, f} \sigma_{i, g}} \cdot \frac{\overline{2 f_{i}} \overline{g_{i}}}{f_{i}^{2}+g_{i}^{2}} \cdot \frac{2 \sigma_{i, f} \sigma_{i, g}}{\sigma_{i, f}^{2}+\sigma_{i, g}^{2}}
$$

$\overline{g_{i}}$ and $\overline{f_{i}}$ defined as the mean value of sub image window for original and enhanced image, $\sigma_{i, f}$ and $\sigma_{i, g}$ is the standard deviation of $i^{\text {th }}$ sub image window for original and enhanced image, $\sigma_{i, f_{g}}$ present the covariance between them. In this paper, window size $5 \times 5$ has been considered.

The 72 different RGB wound images are applied from our wound database to the 16 different filters and find out the best filter based on considering 8 important parameters for improving the image quality. Then we have comparatively analyzed by averaging the all parameters values that shown in Table 1. Lower value of MSE, NAE, and MAE refers that there is very small change between original and filtered image. On the other hands, higher value of PSNR, SNR reports that there is very small change. From Figure 3 to 10, we observe that adaptive median filter is the best alternative for visualization because it provides good value of PSNR (66.23), SNR (58.05), MAE (0.29), NAE (0.01), MD (126.10), MSSIM (1.00), UIQI (0.99) and MSE (3.01) values. The value of MSSIM and UIQI closer to 1 refers the best possible quality of image as well as structural similarity. Midpoint filtering 
Table 1 Quality performance measure of 16 filters considered for study

\begin{tabular}{lrrrrrrrr}
\hline Filters & MSE & SNR & PSNR & NAE & MD & MAE & MSSIM & UIQI \\
\hline Median & 13.09 & 25.12 & 39.21 & 0.02 & 154.16 & 1.03 & 0.98 & 0.99 \\
Mean & 76.20 & 17.01 & 8.58 & 1.72 & 208.21 & 58.52 & 0.43 & 0.48 \\
Average & 17.02 & 21.07 & 36.00 & 0.02 & 100.01 & 1.21 & 0.99 & 0.99 \\
Min & 88.28 & 14.23 & 29.45 & 0.04 & 301.26 & 4.16 & 0.97 & 0.97 \\
M3 & 91.40 & 19.18 & 28.95 & 0.06 & 191.16 & 2.79 & 0.92 & 0.98 \\
Max & 56.25 & 21.02 & 31.46 & 0.05 & 118.42 & 0.30 & 0.98 & 0.97 \\
Log & 92.72 & 6.93 & 7.89 & 0.94 & 254.79 & 62.02 & 0.08 & 0.11 \\
Laplacian & 94.64 & 9.90 & 27.70 & 0.08 & 143.68 & 3.42 & 0.95 & 0.99 \\
Midpoint & 11.34 & 37.93 & 62.67 & 0.00 & 13.47 & 0.04 & 1.00 & 1.00 \\
Gaussian & 33.11 & 36.84 & 46.40 & 0.02 & 45.84 & 1.30 & 1.00 & 0.99 \\
Contra harmonic & 71.13 & 25.02 & 27.03 & 0.07 & 106.79 & 1.40 & 0.90 & 0.94 \\
Adaptive median & $\mathbf{3 . 0 1}$ & $\mathbf{5 8 . 0 5}$ & $\mathbf{6 6 . 2 3}$ & $\mathbf{0 . 0 1}$ & $\mathbf{1 2 6 . 1 0}$ & $\mathbf{0 . 2 9}$ & $\mathbf{1 . 0 0}$ & $\mathbf{0 . 9 9}$ \\
Butter worth & 86.02 & 19.18 & 25.66 & 0.10 & 80.00 & 3.71 & 0.89 & 0.94 \\
Weiner & 60.14 & 24.23 & 31.38 & 0.07 & 69.74 & 2.15 & 0.90 & 0.94 \\
Alpha trim mean & 94.10 & 14.25 & 16.53 & 0.35 & 225.68 & 31.99 & 0.82 & 0.78 \\
Gabor & 65.01 & 15.23 & 19.74 & 0.24 & 138.74 & 22.17 & 0.93 & 0.90 \\
\hline
\end{tabular}

\section{MSE}

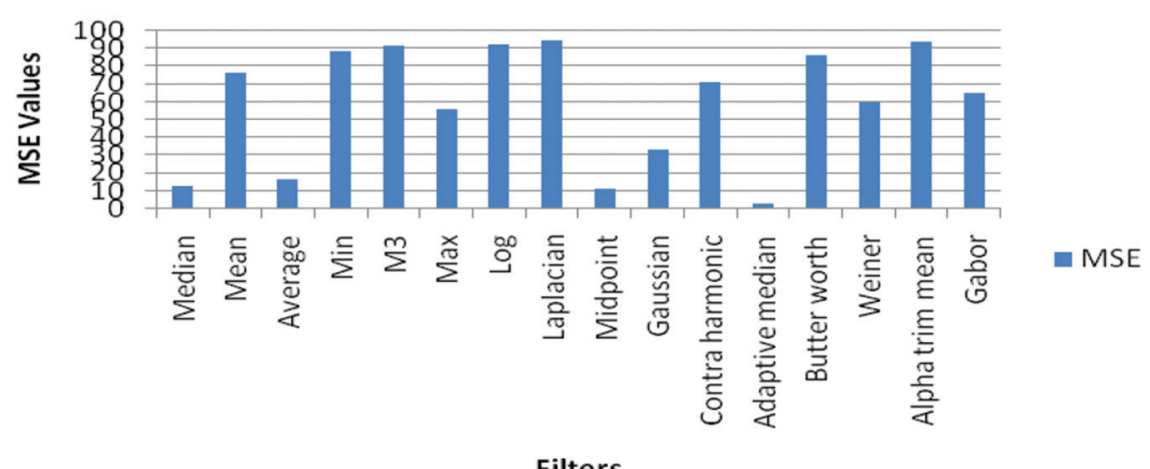

Figure 3 MSE parameter measurement with image quality.

provides good results similar to the Adaptive Median but Midpoint having higher MSE value (11.34) in comparison the adaptive median. High value of MSE means more error from the mean value. This filtering technique improves the segmentation performance with the maintaining proper homogeneity of the image. From all the above discussion, it can be concluded that adaptive median filter provides better visualization after observing the all filtered image results. So any unknown image can be proceed through 


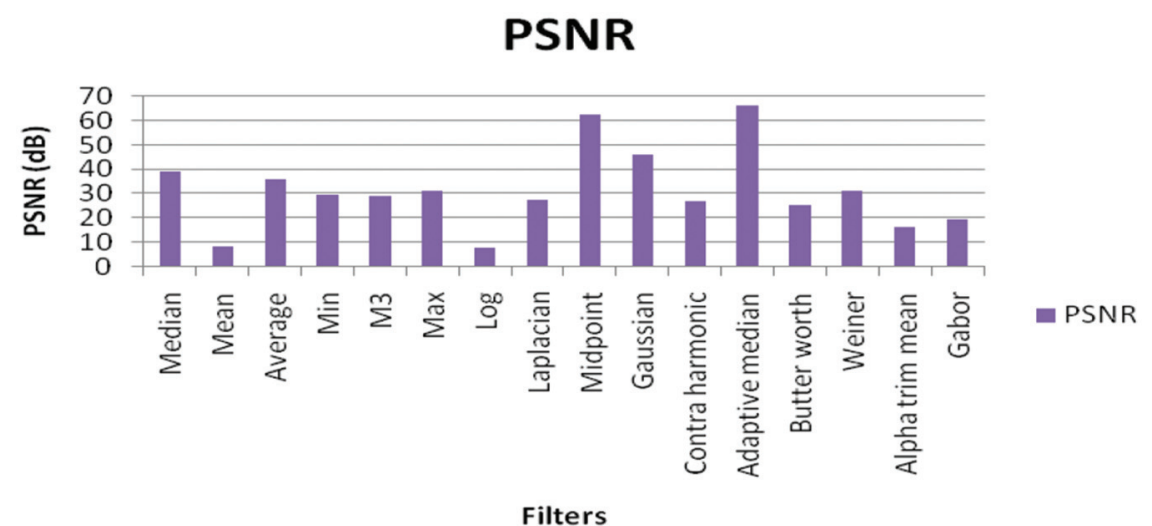

Figure 4 PSNR parameter measurement with image quality.

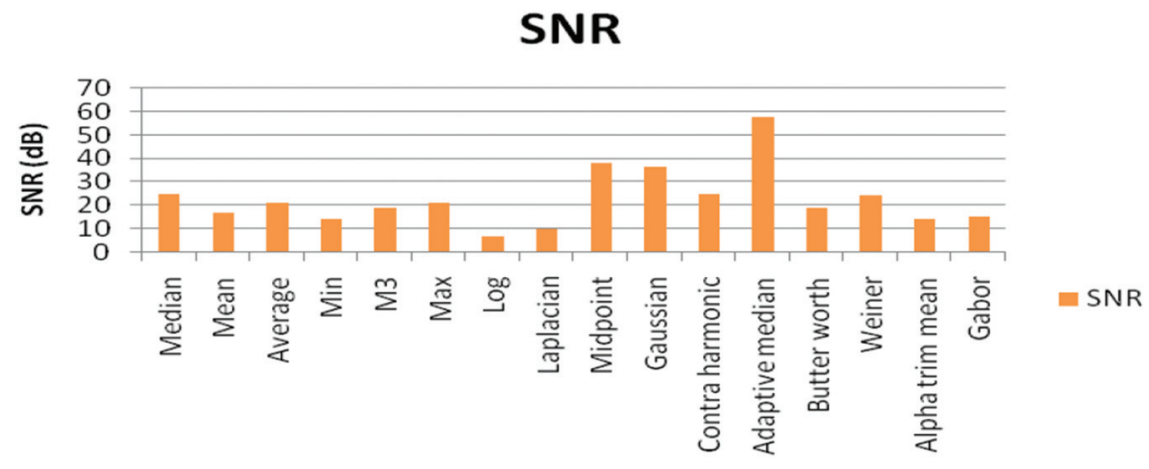

Filters

Figure 5 SNR parameter measurement with image quality.

adaptive median filter and it gives better outcome in pre-processing module. This result has been validated by expert dermatologist. According to expert opinion, sharpness of a CW healing edge is preserved and can be visualize predominantly.

\section{Conclusion}

In this paper, we have discussed about the 16 different filtering techniques for chronic wound analysis under tele-wound network. Furthermore, we have compared all the parametric output for all filtering techniques. The acquired 


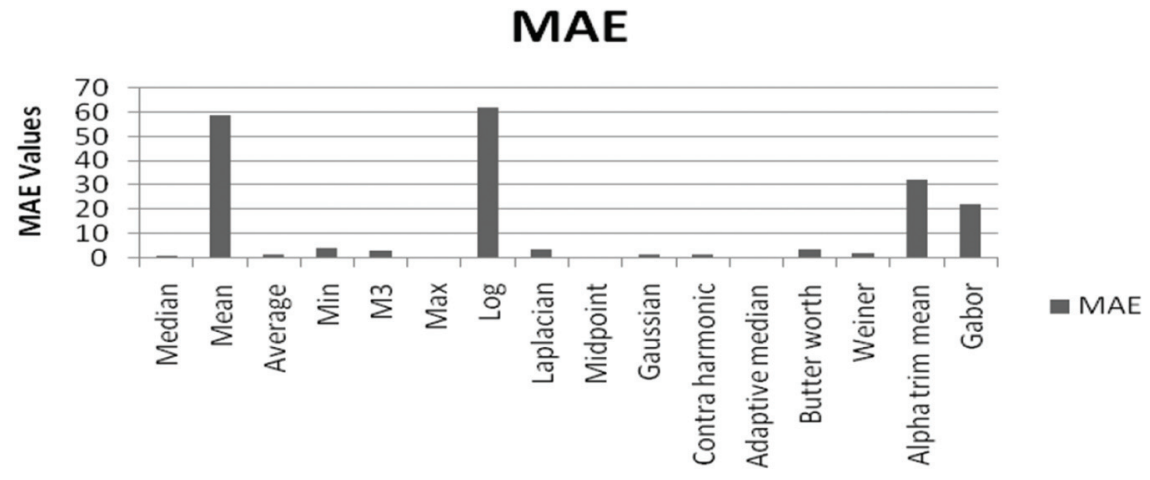

Filters

Figure 6 MAE parameter measurement with image quality.

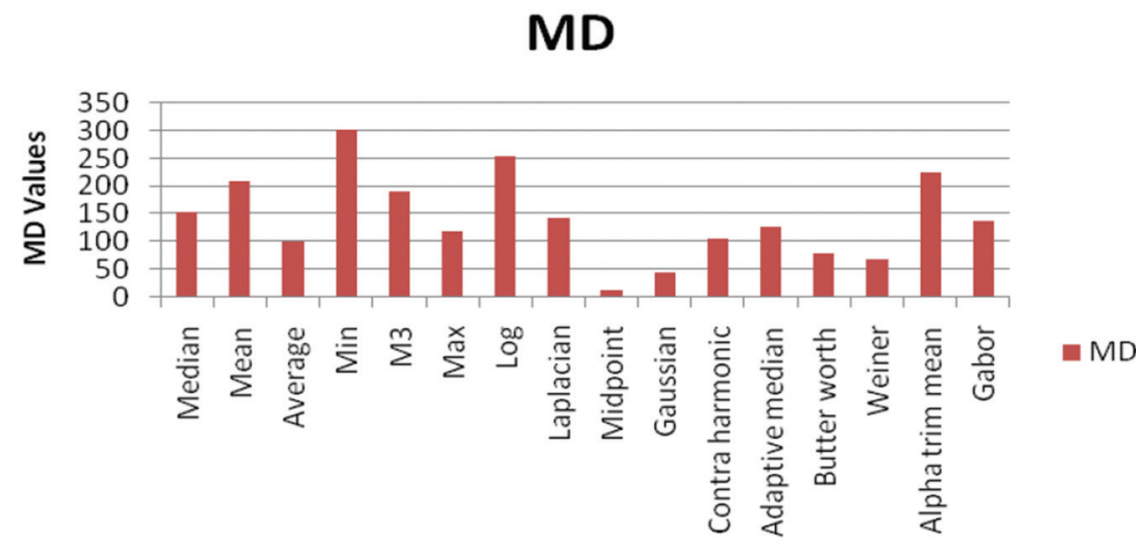

Filters

Figure 7 MD parameter measurement with image quality.

wound images undergo filtering to remove the noise based on the optimal parameter for quality performance analysis. Simulated results have shown that adaptive median filter is better for such noise removal than the other filters. We have presented a comparative study of all filters and selected best filter based on average values of set of all images. Results shows adaptive median provides better performances with respect to high PSNR (66.23) and reduced MSE (3.01), NAE (0.01), MAE (0.29), UIQI (0.99) between original and filtered image. The overall system performances in pre-processing stage would have 


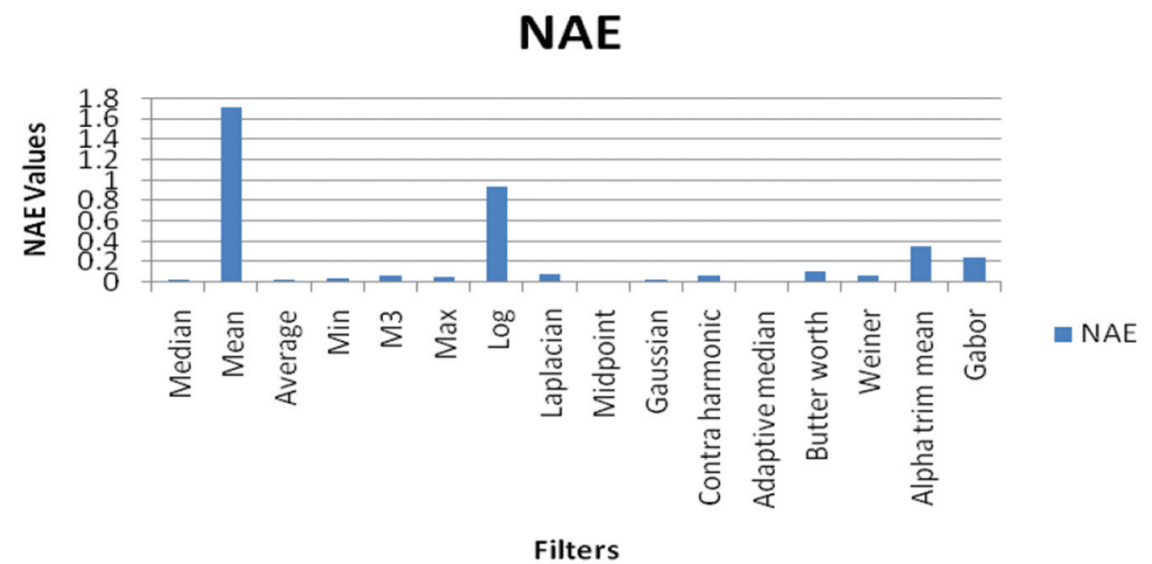

Figure 8 NAE parameter measurement with image quality.

\section{MSSIM}

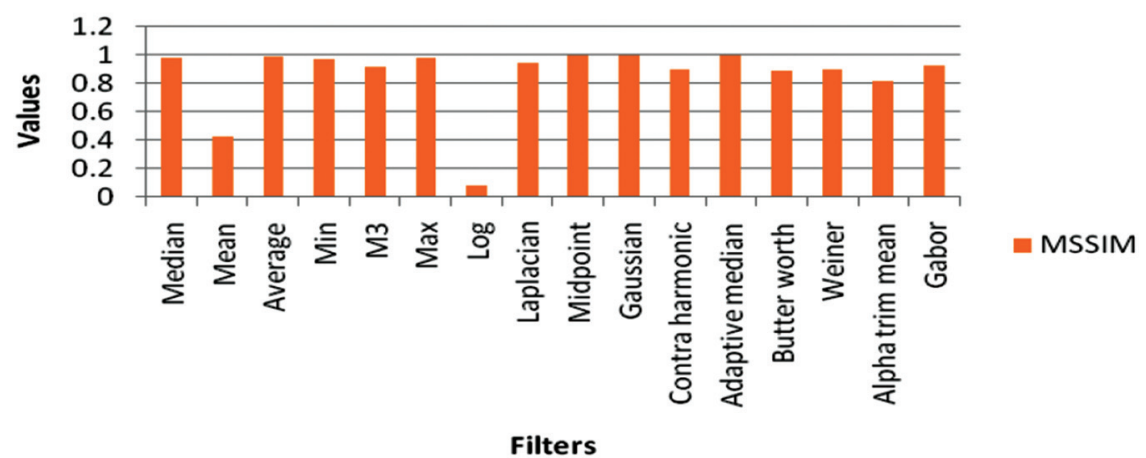

Figure 9 MSSIM parameter measurement with image quality.

been improved using adaptive filtering scheme. Outcome obtained manifests that the system has greater scope of utilization in the field of monitoring and diagnosis of chronic wound through tele-wound network.

\section{Acknowledgment}

The authors would like to acknowledge Dr. Subhas C. Choudhary, Surgeon, Skin Specialist, Jharkhand, India, for his valuable opinion to carry out this work. 


\section{UIQI}

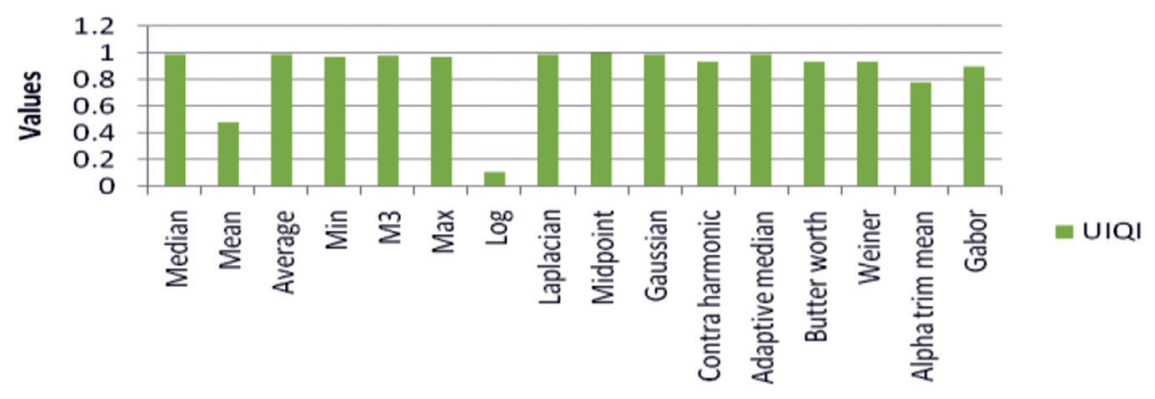

Filters

Figure 10 UIQI parameter measurement with image quality.

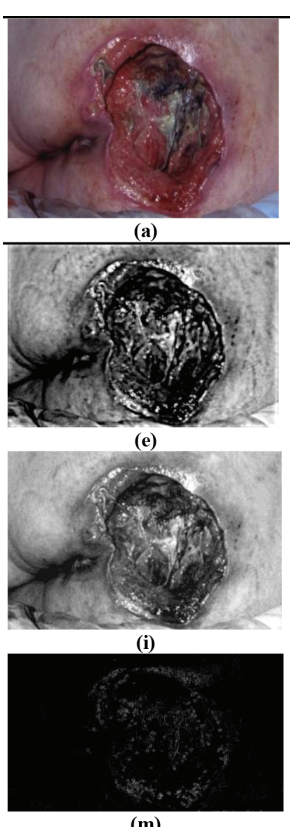

(m)

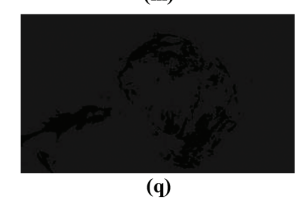

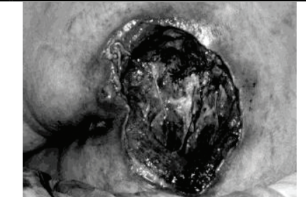

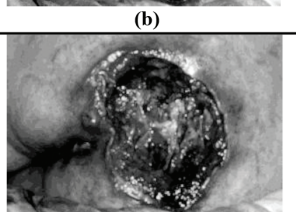

(f)

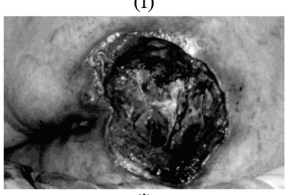

(j)

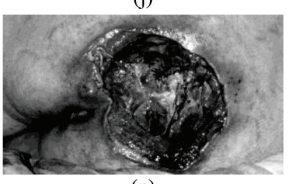

(n)
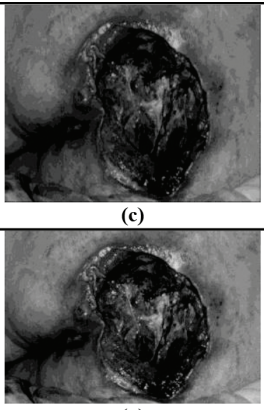

(g)

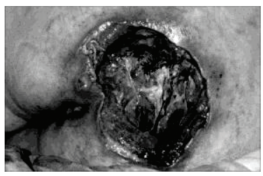

(k)

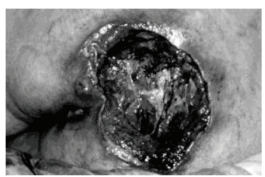

(o)
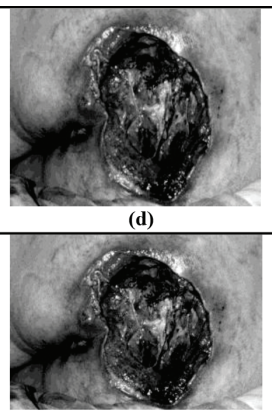

(h)
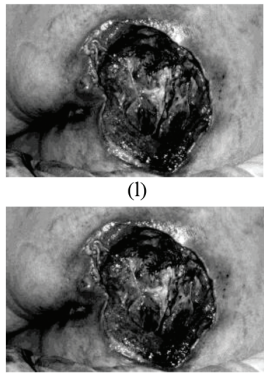

(p)

Figure 11 (a) Original Image, (b) Adaptive median, (c) Alpha Trim mean, (d) Average, (e) Butterworth, (f) Contra harmonic, (g) Gabor, (h) Median, (i) Gaussian, (j) Weiner, (k) Midpoint, (l) Mean, (m) Log, (n) Laplacian, (o) M3, (p) Max, (q) Min. 


\section{References}

[1] Chakraborty C., Gupta B., Ghosh S.K., "Identification of Chronic Wound Status under Tele-Wound Network through Smartphone, IGI Global International Journal of Rough Sets and Data Analysis," Special issue on: Medical Image Mining for Computer-Aided Diagnosis, vol. 2, no. 2, pp. 56-75, 2015.

[2] Chakraborty C., Gupta B., Ghosh S.K., "Tele-wound monitoring through smartphone," IEEE: Int. Conf. on Medical Imaging, m-Health and Emerging Communication Systems (MedCom), pp. 197-201, 2014.

[3] Gonzalez R., Richard W., "Digital Image Processing," Addison-Wesley Publishing Company, 2001.

[4] Wild T., Prinz M., Fortner N., Krois W., Sahora K., Stremitzer S., Hoelzenbein T., "Digital measurement and analysis of wounds based on colour segmentation," European Surgery, pp. 1-6, 2008.

[5] Christos P.L., Takis K., Michalis P., "Evaluation of wound healing process based on texture image analysis," Journal of Biomedical Graphics and Computing, vol. 3, no. 3, pp. 1-13, 2013.

[6] Lei W., Peder C.P., Diane S., Bengisu T., Emmanuel A., "Wound image analysis system for diabetics," Medical Imaging, Proc. of SPIE, vol. 8669, pp. 1-14, 2013.

[7] Barone S., Paoli A., Razionale A.V., "Assessment of chronic wounds by three-dimensional optical imaging based on integrating geometrical, chromatic, and thermal data," Proc. of IMechE, vol. 225, no. 181, pp. 1-14, 2011.

[8] Éderson. A. G. D., Marco A. C., Frade1, Rangaraj M., Rangayyan, Paulo M., Azevedo M., "Segmentation and analysis of the tissue composition of dermatological ulcers," $23^{\text {rd }}$ Canadian Conf. on Electrical and Computer Engineering (CCECE), pp. 1-4, 2010.

[9] Marina K., Ales F., "Segmentation of wounds in the combined colortexture feature space," SPIE, pp. 1-8, 2004.

[10] Dorra N., Yves L., Sylvie T., Jolivot R., Marzani F., "Color and multispectral imaging for wound healing evaluation in the context of a comparative preclinical study," Medical Imaging, pp. 1-10, 2013.

[11] Chakraborty C., Gupta B., Ghosh S., "Mobile metadata assisted community database of chronic wound," Int. Journal of Wound Medicine, Elsevier, vol. 6, pp. 34-42, 2014.

[12] Chakraborty C., Gupta B., Ghosh S.K., "A Review on TelemedicineBased WBAN Framework for Patient Monitoring," Int. Journal of 
Telemedicine and e-Health, Mary Ann Libert inc., vol. 19, no. 8, pp. 619-626, 2013.

[13] Chakraborty C., Gupta B., Ghosh S.K., Das KD, Chakraborty C., "Telemedicine Supported Chronic Wound Tissue Prediction Using Different Classification Approach", Journal of Medical Systems, vol. 40, no. 3, pp. 1-12, 2016.

[14] Arias-Castro E., Donoho D.L., "Does median filtering truly preserve edges better than linear filtering," Annals of Statistics, vol. 37, No. 3, pp. 1172, 2009.

[15] Perona P., Malik J., "Scale-space and edge detection using anisotropic diffusion," IEEE Transactions on Pattern Analysis and Machine Intelligence, vol. 12, No. 7, pp. 629-639, 1990.

[16] Brainard, D.H., Wandell, B.A., "Analysis of the retinex theory of color vision," JOSAA, Vol. 3, pp. 1651-1661, 1986.

[17] Wang, A.C. Bovik, Sheikh H.R., Simoncelli E.P., "Image quality assessment: From error visibility to structural similarity," IEEE Trans. Image Process., vol. 13, no. 4, pp. 600-612, 2004.

[18] Poobal, S., Ravindran, G., "The performance of fractal image compression on different imaging modalities using objective quality measures" International Journal of Engineering Science and Technology (IJEST), vol. 2, pp. 239-246, 2011.

\section{Biographies}

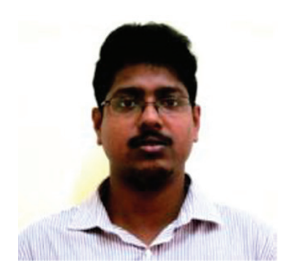

C. Chakraborty received the B.Tech. degree in Electronics and Communication Engineering from Maulana Abul Kalam Azad University of Technology, India in 2006. From 2006 to 2007, he was with the Department of Industrial Engineering and Management, Indian Institute of Technology, Kharagpur, India as a Research Consultant. He received the M.S. degree in Telecommunication Engineering from G. S. Sanyal School of Telecommunications, IIT Kharagpur, India in 2010. He is worked at ICFAI Univ., Agartala, India as Senior Lecturer. Currently $\mathrm{He}$ is working at Birla Institute of Technology, 
Mesra, India as an Assistant Professor. His research interests include Internet of Medical Things, 5G, Telemedicine, and WBAN. He has published more than 25 technical papers in various journals and conferences.

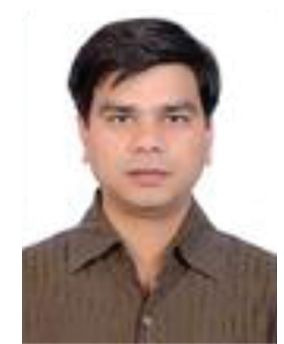

B. Gupta received B.E. and M.Tech. degrees in Electronics Engineering from Govt. Engineering College of Ujjain, Madhya Pradesh, India and Birla Institute of Technology in 2000 and 2003 respectively. The Ph.D. degree he received in the area of Wireless Communication from the Department of Microelectronics and Telecommunication at Univ. of Rome, Tor Vergata, Italy, in 2011. He is a senior member of IEEE and Member of IET. Dr. Gupta worked in Birla Institute of Technology, Mesra, from August 2004 till July, 2016. Presently, he has joined National Institute of Technology, Patna as a Assistant Professor in Electronics and Communication Engineering department. His teaching and research interests include UWB Communication, Energy Efficient MAC for WBAN, Vital Sign Monitoring and WSN. He has published more than 40 technical papers in various journals and conferences. 